\title{
The RANTANPLAN planner: system description
}

\author{
Miquel Bofill, Joan Espasa and Mateu Villaret \\ Departament d'Informàtica, Matemàtica Aplicada i Estadística \\ Universitat de Girona, Spain. \\ E-mail: \{miquel.bofill@udg.edu, joan.espasa@udg.edu,mateu.villaret@udg.edu\}
}

\begin{abstract}
RANTANPLAN is a numeric planning solver that takes advantage of recent advances in SMT. It extends reduction to SAT approaches with an easy and efficient handling of numeric fluents using background theories. In this paper we describe the design choices and features of RANTANPLAN, especially, how numeric reasoning is integrated in the system. We also provide experimental results showing that RANTANPLAN is competitive with existing exact numeric planners.
\end{abstract}

\section{Introduction}

The problem of planning, in its most basic form, consists in finding a sequence of actions that allow to reach a goal state from a given initial state. Although initially considered a deduction problem, it was rapidly seen that it could be addressed by looking at it as a satisfiability (model finding) problem (Kautz and Selman, 1992). Many (incomplete) heuristic methods can be found in the literature to efficiently deal with this problem, most of them oriented towards finding models. Exact methods were ruled out at the beginning due to their inefficiency. However, in (Kautz et al., 1996) it was shown that modern off-the-shelf SAT solvers could be effectively used to solve planning problems. In recent years, the power of SAT technology has been leveraged to planning (Rintanen, 2012), making reduction into SAT competitive with heuristic search methods.

Although a lot of work has been devoted to the encoding of plans in propositional logic, only a few works can be found in the literature on satisfiability based approaches to planning in domains that require numeric reasoning. This is probably due to the difficulty of efficiently handling at the same time numeric constraints and propositional formulas. Among the few works dealing with planning with resources are (Hoffmann, 2003; Kautz and Walser, 1999; Gerevini et al., 2008; Hoffmann et al., 2007). There have also been some works using constraint and logic programming (Dovier et al., 2010; Barták and Toropila, 2010). However, the advances in satisfiability modulo theories (SMT) (Barrett et al., 2009) in the last years make worth considering this alternative. With RANTANPLAN we demonstrate that with SMT one can elegantly handle numeric reasoning inside any PDDL domain, thanks to the integration of various background theories with a SAT solver.

As the number of variables, and hence the search space, rapidly grows with the number of time steps considered, a key idea to improve the performance of SAT-based planners is to consider the possibility of executing several actions at the same time, i.e., the notion of parallel plans. Parallel plans increase the efficiency not only because they allow to reduce the time horizon, but also because it is unnecessary to consider all total orderings of the actions that are performed in parallel. Nevertheless, in SAT-based planning, parallel plans are not intended to represent true parallelism in time, and it is usually required that a sequential plan can be built from a parallel plan in polynomial time. Two main types of parallel plans are considered: $\forall$-step plans, and $\exists$-step plans. In $\forall$-step plans, any ordering of parallel actions must result in a valid sequential plan. In 
$\exists$-step plans, there must exist a total ordering of parallel actions resulting in a valid sequential plan. We refer the reader to (Rintanen et al., 2006) for further details. RANTANPLAN supports $\forall$ and $\exists$-step plans, using various different encodings.

To ensure that a parallel plan is sound, it is necessary that all actions proposed to be executed at the same time do not interfere. Different notions of interference have been defined, some more restrictive, some more relaxed. As far as we know, for efficiency reasons, potential interference between action is always determined statically, i.e., independently of any concrete state, hence in a fairly restrictive way. Moreover, very few works deal with the notion of incompatibility of actions in planning with resources, most of them with rather syntactic or limited semantic approaches (Kautz and Walser, 1999; Fox and Long, 2003; Gerevini et al., 2008). RANTANPLAN incorporates a novel method for determining interference between actions at compile time, using an SMT solver as an oracle.

Summing up, RANTANPLAN is a numeric planner based on planning as satisfiability, which translates PDDL problems into SMT formulas. It supports various types of parallelism, using a novel notion of interference. Experimental results show that it is competitive with other exact numeric planners and strictly better in non-trivial numeric domains.

\section{Related Work}

The pioneering work of LPSAT (Wolfman and Weld, 1999) on planning with resources can indeed be considered one of the precursors of SMT, as the basic ideas of SMT (Boolean abstraction, interaction of a SAT solver with a theory solver, etc.) were already present in it.

A comparison between SAT and SMT based encodings for planning in numeric domains can be found in (Hoffmann et al., 2007). In the SAT approach, the possible values of numeric state variables is approximated, by generating a set of values $D_{t}(v)$ for every numeric variable $v$, so that every value that $v$ can have after $t$ time steps is contained in $D_{t}(v)$. These finite domains then serve as the basis for a fully Boolean encoding, where atoms represent numeric variables taking on particular values. With respect to SMT, where numeric variables and expressions are first class citizens, the authors argue that the expressivity of the SMT language comes at the price of requiring much more complex solvers than for SAT and, for this reason, their SAT-based method is very efficient in domains with tightly constrained resources, where the number of distinct values that a numeric variable can take is small.

Other approaches, related to SMT to some amount as well, have been developed more recently. In (Belouaer and Maris, 2012), a set of encoding rules is defined for spatio-temporal planning, taking SMT as the target formalism. On the other hand, in (Gregory et al., 2012) a modular framework, inspired in the architecture of lazy SMT, is developed for planning with resources.

\section{Preliminaries}

A numeric planning problem is defined as a tuple $\langle V, P, A, I, G\rangle$ where $V$ is a set of numeric variables, $P$ is a set of propositions (or Boolean variables), $A$ is a set of actions, $I$ is the initial state and $G$ is a formula over $V \cup P$ that any goal state must satisfy.

A state is a total assignment to the variables. Actions are formalized as pairs $\langle p, e\rangle$, where $p$ are the preconditions and $e$ the effects. More formally, $p$ is a set of Boolean expressions over $V \cup P$, while $e$ is a set of (conditional) effects of the form $f \Rightarrow d$, where $f$ is a Boolean expression over $V \cup P$ and $d$ is a set of assignments. An assignment is a pair $\langle v, \exp \rangle$, where $v$ is a variable and exp is an expression of the corresponding type. For example, increasing a variable $v$ by one is represented by the pair $\langle v, v+1\rangle$, indicating that $v+1$ is the value that $v$ will hold in the next state. Unconditional effects are represented by setting $f=$ true.

The active effects of an action $a=\langle p, e\rangle$ in a state $s$ are $\cup_{f \Rightarrow d \in e}\{d|s|=f\}$. An action $a=\langle p, e\rangle$ is executable in a given state $s$ if $s=p$ and the active effects of $a$ in state $s$ are consistent. For numeric variables, we restrict to the case where there is only one assigment per variable in the active effects. 


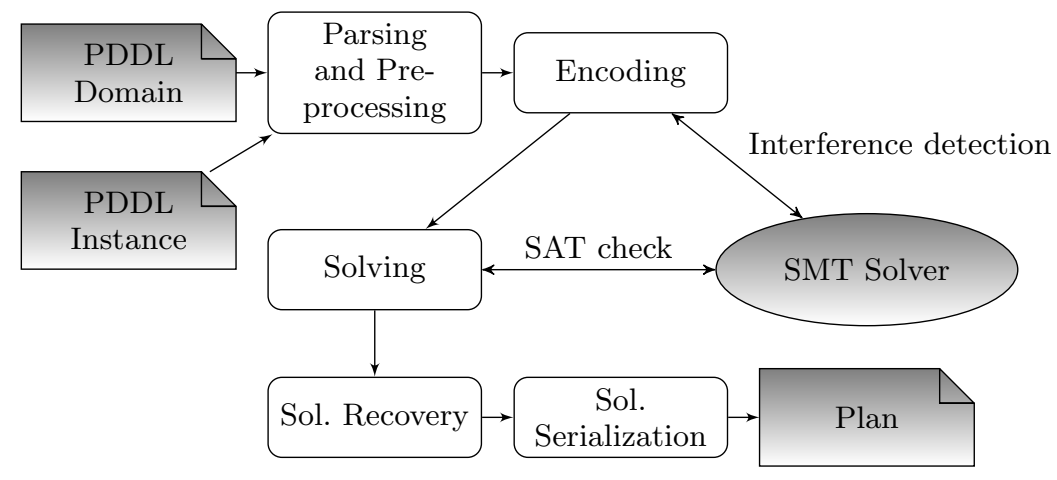

Figure 1: Basic architecture and solving process of the RANTANPLAN solver

The state resulting from executing action $a$ in state $s$ is denoted by $\operatorname{apply}(a, s)=s^{\prime}$. The new state $s^{\prime}$ is defined by assigning new values to the variables according to the active effects, and retaining the values of the variables that are not assigned values by any of the active effects.

A sequential plan of length $n$ for a given planning problem $\langle V, P, A, I, G\rangle$ is a sequence of actions $a_{1} ; a_{2} ; \ldots ; a_{n}$ such that apply $\left(a_{n} \ldots\right.$ apply $\left.\left(a_{2}, \operatorname{apply}\left(a_{1}, I\right)\right) \ldots\right) \models G$.

A parallel plan of length $n$ can be defined similarly to a sequential plan. Instead of having a sequence of actions, we have a sequence of sets of actions $\sigma_{1} ; \sigma_{2} ; \ldots ; \sigma_{n}$ such that $\operatorname{order}\left(\sigma_{1}\right) \oplus$ $\operatorname{order}\left(\sigma_{2}\right) \oplus \cdots \oplus \operatorname{order}\left(\sigma_{n}\right)$ is a sequential plan, where $\operatorname{order}\left(\sigma_{i}\right)$ is an ordering function which transforms the set $\sigma_{i}$ into a sequence of actions, and $\oplus$ denotes the concatenation of sequences. Actions in the same set $\sigma_{i}$ are said to occur in parallel.

The notion of parallelism of a $\forall$-step plan is defined as the possibility of ordering the actions of each set to any total ordering, i.e., no two actions $a, a^{\prime}$ in each $\sigma_{i}$ are interfering (e.g., executing $a$ neither falsifies the precondition of $a^{\prime}$ nor changes any of its active effects, and vice versa).

The $\exists$-step semantics weakens the $\forall$-step requirements, by only requiring the existence of some correct ordering of the actions that results in a valid sequential plan.

In the planning as SAT approach, a planning problem is solved by considering a sequence of formulas $\phi_{0}, \phi_{1}, \phi_{2}, \ldots$, where $\phi_{i}$ encodes the feasibility of a plan that allows to reach a goal state from the initial state in $i$ steps. The solving procedure proceeds by testing the satisfiability of $\phi_{0}, \phi_{1}, \phi_{2}$, and so on, until a satisfiable formula $\phi_{n}$ is found. It is a matter of the encoding whether one or various (non interfering) actions are executed at each step.

\section{Framework and System Architecture}

RANTANPLAN supports a fragment of PDDL which is close to general numeric PDDL 2.1, excluding the temporal extensions and metric optimizations. With respect to numeric effects, we consider $\operatorname{assign}(x, \exp )$, increase $(x, \exp )$ and decrease $(x, \exp )$, where exp is any constant expression over linear integer (or real) arithmetic. With respect to preconditions and conditions of numeric effects, we assume that the restrictions imposed on numeric fluents take the form of any closed formula over linear integer (or real) arithmetic.

The structure of the RANTANPLAN system is represented in Figure 1. To encode the formulas $\phi_{0}, \phi_{1}, \phi_{2}, \ldots$, one of the two encodings described in the following sections (QF_LIA or QF_UFLIA) is carried out, transforming the PDDL problem to a pure SMT problem. Then the problem is iteratively solved, using the chosen SMT Solver as a black box.

A key aspect of the planner is the detection of interferences between parallel actions at compile time, by means of calls to a SMT Solver. In case the user demands a parallel plan, a disabling graph is computed. By disabling graph we refer to a directed graph, where nodes are the grounded 
actions from the planning problem and an edge exists from action $a$ to action $a^{\prime}$ if the execution of $a$ can affect $a^{\prime}$ (forbid its execution or change its active effects) (Rintanen et al., 2006). This graph is used, depending on the notion of parallelism chosen, to encode the necessary constraints restricting which actions can be carried out at the same time step. In particular, the solver supports:

- A sequential encoding, achieved by using an at least one and an at most one constraint.

- The $\forall$-step semantics, using the quadratic encoding in (Rintanen et al., 2006)

- The $\exists$-step semantics, with two encodings. Using the quadratic encoding in (Rintanen et al., 2006), and a linear-size encoding based on a fixed ordering of operators, also in (Rintanen et al., 2006).

The system supports solving via API or plain text file using the Yices SMT solver and the Z3 SMT solver. Once a solution has been found, then it is finally retrieved and serialized. In the following subsections, the relevant aspects of the RANTANPLAN solver are explained in more detail.

\subsection{QF_LIA Encoding}

Numeric planning problems with linear integer arithmetic expressions naturally fall into the QF_LIA logic. In the SMT-LIB standard, QF_LIA stands for the logic of Quantifier-Free Boolean formulas, with Linear Integer Arithmetic constraints. This logic has a good compromise between expressivity and performance, and is the natural choice for this problem. We generalized Rintanen's (Rintanen, 2012) encoding of planning as SAT to include numeric variables as follows.

For each time step, every ground instance of a PDDL predicate and action is mapped to a Boolean variable, and every ground instance of a PDDL function is mapped to an integer variable. For instance, a predicate stating the position of an aircraft such as at(?a - aircraft, ?c city), with three cities $c 1, c 2$ and $c 3$, and two planes $\mathrm{p} 1$ and $\mathrm{p} 2$, will result into six ground instances at $(\mathrm{p} 1, \mathrm{c} 1), \ldots$, at $(\mathrm{p} 2, \mathrm{c} 3)$, that will be mapped to six Boolean variables $a t_{p 1, c 1}^{t}$, $\ldots, a t_{p 2, c 3}^{t}$ for each time step $t$. Following the same example, being at(?o - aircraft) city an object fluent, the mapping would result into two integer variables $a t_{p 1}$, $a t_{p 2}$ with the domains being the possible cities $c 1, c 2$ and c3 (these are internally mapped into three distinct integers). Note that thanks to the SMT language, we can get a more compact encoding of states in the presence of object fluents than using a plain SAT approach. The Boolean variables resulting from actions will be used to denote what action is executed at each time step, and with which parameters. The Boolean and integer variables resulting from grounding the predicates and functions, respectively, will constitute the state variables. A superscript $t$ is used to differentiate the variables at each time step.

Given a formula $\phi$, by $\phi^{t}$ we denote the same formula $\phi$ where all integer variables $x$ have been replaced by $x^{t}$. For the case of assignments, we define:

$$
\begin{gathered}
\langle x, \text { true }\rangle^{t} \stackrel{\text { def }}{=} x^{t} \\
\langle x, \text { false }\rangle^{t} \stackrel{\text { def }}{=} \neg x^{t} \\
\langle x, k\rangle^{t} \stackrel{\text { def }}{=}\left(x^{t}=k\right) \\
\langle x, x+k\rangle^{t} \stackrel{\text { def }}{=}\left(x^{t}=x^{t-1}+k\right) \\
\langle x, x-k\rangle^{t} \stackrel{\text { def }}{=}\left(x^{t}=x^{t-1}-k\right)
\end{gathered}
$$


For each ground ${ }^{1}$ action $a=\langle p, e\rangle$, we have the following constraints. First, its execution during time step $t$ implies that its precondition is met:

$$
a^{t} \rightarrow p^{t} \quad \forall a=\langle p, e\rangle \in A
$$

Also, each of its conditional effects will hold at the next time step if the corresponding condition holds:

$$
\left(a^{t} \wedge f^{t}\right) \rightarrow d^{t+1} \quad \forall a=\langle p, e\rangle \in A, \forall f \Rightarrow d \in e
$$

Here we view sets $d$ of literals as conjunctions of literals. Recall also that unconditional effects will have true as condition $f$. Second, we need explanatory axioms to express the reason of a change in state variables. For each variable $x$ in $V \cup P$ :

$$
x^{t} \neq x^{t+1} \rightarrow \bigvee_{a=\langle p, e\rangle \in A}\left(a^{t} \wedge\left(E P C_{x}(a)\right)^{t}\right)
$$

where, given an action $a=\langle p, e\rangle$ and a variable $x$,

$$
E P C_{x}(a)=\bigvee_{f \Rightarrow d \in e}\{f \mid d \text { contains an assignment for } x\}
$$

that is, the effect precondition for the modification of $x$ in action $a$, where the empty disjunction is defined as false. For Boolean variables, the expression $x^{t} \neq x^{t+1}$ can be written as $\left(x^{t} \wedge \neg x^{t+1}\right) \vee$ $\left(\neg x^{t} \wedge x^{t+1}\right)$. These constraints have to be complemented depending on the parallelism we wish.

\subsection{Interference Between Actions}

As said in the introduction, a key concept in parallel plans is the notion of interference between actions. This issue has been carefully considered by Rintanen et al. (Rintanen et al., 2006) in the setting of planning as SAT. Given a disabling graph, where an edge exists from action $a$ to action $a^{\prime}$ if the execution of $a$ can affect $a^{\prime}$, we know for example that the simultaneous execution of all actions pertaining to a strongly connected component is not possible, as given all possible orderings of actions, all of them contain a cycle (and thus they cannot be serialized). Note that acyclicity is a sufficient but not necessary condition for a set of actions to be executable in some order, since disabling graphs are computed independently of any state.

In (Rintanen et al., 2006), an action $a_{1}$ is defined to affect another action $a_{2}$ if $a_{1}$ may prevent the execution of $a_{2}$ or change its active effects, and two actions $a_{1}$ and $a_{2}$ are considered to interfere if $a_{1}$ affects $a_{2}$ or $a_{2}$ affects $a_{1}$. In $\forall$-step plans, where all possible serializations must be valid, no two interfering actions can occur in parallel. In the more relaxed notion of parallelism of $\exists$-step plans, where it is only required that no action affects a later one in some total ordering, often much more parallelism is allowed in practice. For efficiency reasons, typically syntactic (rather than semantic) restrictions are imposed on parallel actions. For example, in (Rintanen et al., 2006), where only Boolean variables are considered, $a_{1}=\left\langle p_{1}, e_{1}\right\rangle$ is determined to affect $a_{2}=\left\langle p_{2}, e_{2}\right\rangle$ if, for some variable $a$,

1. $a$ is set to true in $d_{1}$ for some $f_{1} \Rightarrow d_{1} \in e_{1}$, and $a$ occurs negatively in $p_{2}$ or occurs in $f_{2}$ for some $f_{2} \Rightarrow d_{2} \in e_{2}$, or

2. $a$ is set to false in $d_{1}$ for some $f_{1} \Rightarrow d_{1} \in e_{1}$, and $a$ occurs positively in $p_{2}$ or occurs in $f_{2}$ for some $f_{2} \Rightarrow d_{2} \in e_{2}$.

That is, $a_{1}$ affects $a_{2}$ if $a_{1}$ can impede the execution of $a_{2}$, or change its effects. Note that this is not a symmetric relation.

${ }^{1} \mathrm{By}$ a ground action $\langle p, e\rangle$ we refer to an action where $p$ and $e$ are built on the state variables that result from grounding a PDDL model, as explained above. 
This is a fully syntactic check which can be used to establish sufficient although not necessary conditions for finding serializable parallel plans. We can observe that interference between effects is not considered. This is because, in the case two actions have contradictory effects, any formula encoding a plan with those two actions running in parallel will become unsatisfiable.

The previous approach could be naively generalized to the case of numeric variables as follows: an action $a_{1}=\left\langle p_{1}, e_{1}\right\rangle$ affects an action $a_{2}=\left\langle p_{2}, e_{2}\right\rangle$ if, for some variable $x, x$ is modified in $d_{1}$ for some $f_{1} \Rightarrow d_{1} \in e_{1}$, and $x$ occurs in $p_{2}$ or occurs in $f_{2}$ for some $f_{2} \Rightarrow d_{2} \in e_{2}$.

Performing only syntactic checks like the previous seems too much restrictive for numeric variables, even in the case that we determine interference at compile time, i.e., independently of any concrete state. For this reason, we propose a new idea ${ }^{2}$, which is to use SMT technology to perform semantic checks of interference at compile time, in order to increase the amount of parallelization of numeric plans.

Our method is independent of any test suite and does not require any special purpose algorithm, as it relies on encoding the possible interference situations between pairs of actions as SMT formulas and checking their satisfiability, by calling an SMT solver, at compile time. For example, an important difference with the purely syntactic definition of interference of (Rintanen et al., 2006) is that we include the preconditions of the actions in the semantic checks. More precisely, two actions can occur in parallel only if their preconditions can be satisfied simultaneously, regardless of the variables they contain. This way, we are able to avoid many "false positive" interference relationships.

All in all, we obtain a much more fine-grained notion of interference, that we will see how it helps us to increase the parallelization of actions. Note that the interference relationships determined semantically will always be a subset of the interference relationships determined syntactically. Interestingly, we will be using an SMT solver both at compile time, as an oracle to predict interference relationships, and at solving time. For efficiency reasons, to perform the interference checks we do not consider grounded actions, but the original actions in the PDDL model. Since now actions are not instantiated, we need to unify the parameters of the same type in the actions for which we check interference.

Imagine we have two actions, say move(?d - ship ?a ?b - location) and dock(?e - ship ?c - location). We will be interested to know, for example, if the actions interfere in the case that ?d and ?e are the same ship. Or in the case that locations ?a and ?c are the same, etc. We will have to check all the possible combinations of equalities and disequalities between the parameters. These combinations are all the possible partitions of the sets formed by all the parameters of the two considered actions grouped by its most general declared type. Once the set partitions have been generated for each set of parameters, each parameter is substituted by an integer. When we intent for two parameters to be equal (i.e. they are in the same partition), we substitute them for the same integer, and by different integers when we want them to be different. Finally, the formulas encoding the incompatibility between the actions are checked for satisfiability.

These consistency checks can be done in a reasonable time with an SMT solver, and the amount of parallelism achieved is significantly higher than with syntactic approaches. To illustrate the situations where our new notion of interference (thoroughly explained in (Bofill et al., 2016)) is especially accurate, consider the following example. The Planes domain in Figure 2 consists in transporting people between cities using planes. Each plane has a limited number of seats and a given fuel capacity. We focus on the fly and board actions. A plane can only fly if it is transporting somebody and it has enough fuel to reach its destination, and boarding is limited by seat availability.

The syntactic notion of interference would determine interference between fly and board, since board modifies the onboard function (number of passengers) and fly checks the value of this function in its precondition. On the contrary, with the semantic technique, we would find out that there is no interference at all, since it is impossible that the preconditions of board and fly

${ }^{2} \mathrm{~A}$ full work devoted to this interference notion is currently submitted for publication. 


\section{Extension: QF_UFLIA Encoding}

As the previously introduced QF LIA encodings grows considerably with the time horizon, to the point of getting unmanageable instances, we started to develop a more compact encoding, using the theory of uninterpreted functions to express predicates, functions and actions. This encoding is reminiscent of the lifted causal encodings in (Kautz et al., 1996).

In the SMT-LIB standard, QF_UFLIA stands for the logic of Quantifier-Free Boolean formulas, with Linear Integer Arithmetic constraints and Uninterpreted Functions. Uninterpreted functions have no other property than its name and arity, and are only subject to the following axiom: $x_{1}=x_{1}^{\prime} \wedge \cdots \wedge x_{n}=x_{n}^{\prime} \rightarrow f\left(x_{1}, \ldots, x_{n}\right)=f\left(x_{1}^{\prime}, \ldots, x_{n}^{\prime}\right)$.

The encoding goes as follows. Every defined object in the problem is mapped to an integer. For each function, predicate and action, an uninterpreted function is declared, with each parameter being declared as an integer. Also, a new integer parameter is added to each of them, representing a time step. Uninterpreted functions corresponding to predicates and actions return a Boolean value, whilst the ones for functions return an integer value. Moreover, for each action, parameter and time step, a new integer variable is defined, representing the value of that parameter in the action if executed at the corresponding time step.

For example, the Boolean function $\varphi_{a}\left(x_{a, 1}^{t}, \ldots, x_{a, n}^{t}, t\right)$ determines whether action $a$ with parameters $x_{a, 1}^{t}, \ldots, x_{a, n}^{t}$ is executed at time step $t$. The parameter $t$ is a constant, which is shared between all uninterpreted functions for the actions, predicates and functions in the same time step. Contrarily, $x_{a, 1}^{t}, \ldots, x_{a, n}^{t}$ are variables with finite domains, and constraints are added to restrict their possible values. Regarding predicates and functions, no new variables are defined, since their arguments will be either constants or variables occurring in some action.

We remark that, in this new setting, a state is defined by the value of the uninterpreted functions corresponding to predicates and functions, for a given value of their arguments. Equations (1) and (2) of the QF LIA encoding are generalized here as:

$$
\begin{array}{lll}
\varphi_{a}\left(x_{a, 1}^{t}, \ldots, x_{a, n}^{t}, t\right) \rightarrow p^{t} & \forall a=\langle p, e\rangle \in A \\
\varphi_{a}\left(x_{a, 1}^{t}, \ldots, x_{a, n}^{t}, t\right) \wedge f^{t} \rightarrow d^{t+1} & & \forall a=\langle p, e\rangle \in A, \forall\langle f, d\rangle \in e
\end{array}
$$

Note that this results in a much more compact encoding than if we restrict to QF LIA, since here we are using variables as arguments of functions, and it is the SMT solver who is in charge of guessing the concrete values of the parameters of the executed actions. The considered set of actions $A$ is now parametrized, and hence similar to that of PDDL, with actions like fly $(x, y, z)$, instead of grounded actions like $f y_{p 1, c 1, c 1}, f l y_{p 1, c 1, c 2}$, etc. Equation (3) is generalized as:

$$
\begin{aligned}
& \varphi_{h}\left(c_{h, 1}, \ldots, c_{h, n}, t\right) \neq \varphi_{g}\left(c_{h, 1}, \ldots, c_{h, n}, t+1\right) \rightarrow \\
& \bigvee_{a \in \text { touch }(g)}\left(\varphi_{a}\left(x_{a, 1}^{t}, \ldots, x_{a, m}^{t}, t\right)\right. \\
& i \in 1 . . n, j \in 1 . . m \\
& \text { name }(h, i)=\operatorname{name}(a, j) \\
& \forall h \in H, \forall c_{h, 1}, \ldots, c_{h, n} \in S_{1} \times \cdots \times S_{n}
\end{aligned}
$$

where $H$ is the set of predicates and functions, $\operatorname{touch}(h)$ is the set of actions that may modify $h$, $S_{i}$ is the domain of the $i$-th argument of $\varphi_{h}$, and name $(h, k)$ is the name in the PDDL model of the $k$-th argument of the functor $h$. To help the reader understand the formula, we provide an example. Suppose we have the following simple PDDL problem:

- objects: A,B - truck, L1,L2,L3 - loc

- predicate: at(?t - truck, ?1 - loc)

- actions: travel(?t - truck, ?from ?to - loc) and refuel(?x - truck, ?where - loc)

- function: fuel(?t - truck) - number 
Table 1 Time in seconds followed by the number of parallel steps of the plan found between parentheses, for each instance. TO stands for time out and MO for memory out. Cutoff set to 3600 seconds. The Time and Edges columns show the reduction in time and edges of the disabling graph, respectively, when using the semantic approach. Instances where all approaches timed out are omitted.

(a) Results of the Depots domain

\begin{tabular}{rrrrr}
\hline $\mathrm{n}$ & Syntactic & Semantic & Time & Edges \\
\hline 1 & $4.1(6)$ & $2.8(6)$ & $31.4 \%$ & $41.7 \%$ \\
2 & $32.0(9)$ & $18.3(8)$ & $42.8 \%$ & $44.2 \%$ \\
3 & $166.9(13)$ & $108.9(13)$ & $34.8 \%$ & $44.9 \%$ \\
4 & $438.3(14)$ & $323.0(14)$ & $26.3 \%$ & $45.1 \%$ \\
5 & TO (8) & TO (17) & - & $45.1 \%$ \\
6 & TO (-) & MO (1) & - & - \\
7 & $188.1(10)$ & $131.0(10)$ & $30.4 \%$ & $44.0 \%$ \\
8 & MO (3) & MO (10) & - & $44.5 \%$ \\
\hline
\end{tabular}

(c) Results of the Zenotravel domain

\begin{tabular}{lrrrr}
\hline $\mathrm{n}$ & Syntactic & Semantic & Time & Edges \\
\hline 1 & $0.0(0)$ & $0.0(0)$ & $35.3 \%$ & $76.3 \%$ \\
2 & $0.1(3)$ & $0.0(3)$ & $23 \%$ & $74.3 \%$ \\
3 & $0.2(3)$ & $0.1(3)$ & $34.6 \%$ & $66 \%$ \\
4 & $0.3(4)$ & $0.1(4)$ & $43.5 \%$ & $66.2 \%$ \\
5 & $0.5(4)$ & $0.3(4)$ & $38.9 \%$ & $71.5 \%$ \\
6 & $0.8(6)$ & $0.5(6)$ & $43.5 \%$ & $72.1 \%$ \\
7 & $0.8(5)$ & $0.4(5)$ & $47 \%$ & $72.6 \%$ \\
8 & $2.8(5)$ & $1.7(5)$ & $38.5 \%$ & $68.3 \%$ \\
9 & $26.5(8)$ & $31.0(8)$ & $-16.9 \%$ & $69.6 \%$ \\
10 & $41.6(8)$ & $61.9(8)$ & $-48.7 \%$ & $70.7 \%$ \\
11 & $7.1(6)$ & $4.5(6)$ & $37.2 \%$ & $69 \%$ \\
12 & $105.8(7)$ & $95.1(7)$ & $10.1 \%$ & $70.4 \%$ \\
13 & $1288.3(9)$ & $1291.5(9)$ & $-0.2 \%$ & $72.6 \%$ \\
\hline
\end{tabular}

(b) Results of the Planes domain

\begin{tabular}{rrrrr}
\hline $\mathrm{n}$ & Syntactic & Semantic & Time & Edges \\
\hline 1 & $1.0(13)$ & $0.3(10)$ & $71.5 \%$ & $84.5 \%$ \\
2 & $6.0(16)$ & $1.1(12)$ & $81.2 \%$ & $84.5 \%$ \\
3 & $49.9(18)$ & $8.3(13)$ & $83.4 \%$ & $86.5 \%$ \\
4 & $431.1(21)$ & $40.0(15)$ & $90.7 \%$ & $86.5 \%$ \\
5 & $117.2(20)$ & $27.0(15)$ & $77.0 \%$ & $86.1 \%$ \\
6 & $1294.6(23)$ & $193.3(18)$ & $85.1 \%$ & $86.1 \%$ \\
7 & $621.9(21)$ & $70.9(16)$ & $88.6 \%$ & $85.8 \%$ \\
8 & $834.2(22)$ & $105.7(17)$ & $87.3 \%$ & $85.8 \%$ \\
9 & TO (23) & $2889.1(20)$ & - & $88.0 \%$ \\
\hline
\end{tabular}

(d) Results of the Petrobras Domain

\begin{tabular}{rrrrr}
\hline $\mathrm{n}$ & Syntactic & Semantic & Time & Edges \\
\hline 1 & $14.7(3)$ & $8.8(3)$ & $40.7 \%$ & $50.4 \%$ \\
2 & $19.3(4)$ & $11.2(4)$ & $42.2 \%$ & $51.8 \%$ \\
3 & $24.6(5)$ & $14.0(5)$ & $43.2 \%$ & $53.2 \%$ \\
4 & $47.0(8)$ & $28.2(8)$ & $40.1 \%$ & $54.5 \%$ \\
5 & $74.9(9)$ & $59.5(9)$ & $20.5 \%$ & $55.8 \%$ \\
6 & $133.9(10)$ & $108.7(10)$ & $18.8 \%$ & $57.1 \%$ \\
7 & $700.1(13)$ & $475.1(13)$ & $32.1 \%$ & $58.3 \%$ \\
8 & $833.4(13)$ & $800.0(13)$ & $4.0 \%$ & $59.5 \%$ \\
\hline
\end{tabular}

but its performance decreases in more complex numeric domains like Petrobras and Planes, where the range of possible values for numeric fluents tends to grow. It can be seen that on the Planes domain, containing only a few non-trivial numeric constraints, classical approaches (Syntactic and NumReach) tend to be overly restrictive with respect to incompatibility between actions. In most instances it can be observed an important gap between the number of time steps needed to find a valid plan by NumReach and our semantic approach. This is also generally reflected in terms of solving time.

Table 3 lists the total number of instances of each family, the number of instances solved by NumReach/SAT, NumReach/SMT, and the presented semantic approach. It also gives the number of accumulated parallel time steps used to reach a valid plan on the commonly solved instances by NumReach/SMT and the two methods implemented in RANTANPLAN. Finally, the other columns show the averaged solving time reduction and disabling graph edge reduction on the solved instances. Even in domains that maintain the same number of time steps, the reduced disabling graphs make solving times notably smaller.

Note that the amount of parallelism in RANTANPLAN is notable. With respect to the number of steps, RANTANPLAN is strictly more parallel than NumReach/SAT and NumReach/SMT in nearly all instances.

The only domain where the RANTANPLAN planner is not competitive is the Depots domain. It is obvious that the reachability approach of NumReach is more adequate for this domain. 
Table 2 Time in seconds followed by the number of parallel steps of the plan found between parentheses, for each instance. TO stands for time out and MO for memory out. Cutoff set to 3600 seconds. Instances where all systems timed out are omitted.

(a) Results of the Depots domain

\begin{tabular}{lrrr}
\hline $\mathrm{n}$ & NReach/SAT & NReach/SMT & Semantic \\
\hline 1 & $0.0(6)$ & $1.5(6)$ & $2.8(6)$ \\
2 & $0.5(9)$ & $8.4(9)$ & $18.3(8)$ \\
3 & $5.7(13)$ & $43.1(13)$ & $108.9(13)$ \\
4 & $10.1(15)$ & $134.7(15)$ & $323.0(14)$ \\
7 & $2.5(11)$ & $35.1(11)$ & $131.0(10)$ \\
8 & TO (-) & $362.7(15)$ & MO (10) \\
10 & $4.8(11)$ & $101.2(11)$ & MO (-) \\
13 & $2.9(10)$ & $96.3(10)$ & TO (-) \\
14 & $25.1(16)$ & $1650.0(13)$ & TO (-) \\
16 & $2.2(9)$ & $118.8(9)$ & TO (-) \\
17 & $6.8(8)$ & $313.2(8)$ & TO (-) \\
19 & $18.1(11)$ & $849.4(11)$ & TO (-) \\
\hline
\end{tabular}

(c) Results of the Petrobras domain (b) Results of the Zenotravel domain

\begin{tabular}{lrrr}
\hline $\mathrm{n}$ & NReach/SAT & NReach/SMT & Semantic \\
\hline 1 & $0.0(2)$ & $0.2(2)$ & $0.0(0)$ \\
2 & $0.0(7)$ & $1.5(7)$ & $0.0(3)$ \\
3 & $0.1(6)$ & $3.7(6)$ & $0.1(3)$ \\
4 & $0.0(6)$ & $2.4(6)$ & $0.1(4)$ \\
5 & $0.1(7)$ & $7.0(7)$ & $0.3(4)$ \\
6 & $0.0(7)$ & $4.2(7)$ & $0.5(6)$ \\
7 & $0.1(8)$ & $9.1(8)$ & $0.4(5)$ \\
8 & $0.4(7)$ & $8.1(7)$ & $1.7(5)$ \\
9 & $0.3(9)$ & $18.1(9)$ & $31(8)$ \\
10 & $0.7(9)$ & $24.2(9)$ & $61.9(8)$ \\
11 & $3.5(8)$ & $18.4(8)$ & $4.5(6)$ \\
12 & $3.8(10)$ & $99.6(10)$ & $95.1(7)$ \\
13 & $22.2(11)$ & $555.6(11)$ & $1291.5(9)$ \\
14 & TO (-) & $537.4(9)$ & TO (7) \\
\hline
\end{tabular}

(d) Results of the Planes domain

\begin{tabular}{|c|c|c|c|c|c|c|c|}
\hline $\mathrm{n}$ & NReach/SAT & NReach/SMT & Semantic & $\mathrm{n}$ & NReach/SAT & NReach/SMT & Semantic \\
\hline 1 & $0.4(6)$ & $39.8(6)$ & $8.8(3)$ & 1 & TO (-) & $36.4(15)$ & $0.3(10)$ \\
\hline 2 & $9.8(9)$ & $56.4(6)$ & $11.2(4)$ & 2 & $3.3(18)$ & $37.9(18)$ & $1.1(12)$ \\
\hline 3 & $17.8(10)$ & $93.9(7)$ & $14.0(5)$ & 3 & TO (-) & $229.9(20)$ & $8.3(13)$ \\
\hline 4 & $118.3(11)$ & $256.5(9)$ & $28.2(8)$ & 4 & $4.4(22)$ & $632.0(23)$ & $40.0(15)$ \\
\hline 5 & $317.9(14)$ & $312.3(9)$ & $59.5(9)$ & 5 & TO (-) & $768.4(22)$ & $27.0(15)$ \\
\hline 6 & $325.4(14)$ & $277.2(9)$ & $108.7(10)$ & 6 & TO (-) & $1183.7(25)$ & $193.3(18)$ \\
\hline 7 & TO (-) & 818.1 (11) & $475.1(13)$ & 7 & TO (-) & $1241.2(23)$ & $70.9(16)$ \\
\hline \multirow[t]{2}{*}{8} & TO (-) & $2753.6(12)$ & $800.0(13)$ & 8 & $5.0(24)$ & $1278.2(24)$ & $105.7(17)$ \\
\hline & & & & $\begin{array}{l}9 \\
12\end{array}$ & $\begin{array}{r}\text { TO (-) } \\
15.5(21)\end{array}$ & $\begin{array}{l}\text { TO (-) } \\
\text { TO (-) }\end{array}$ & $\begin{array}{r}2889.1(20) \\
\text { TO }(19)\end{array}$ \\
\hline
\end{tabular}

Table 3 Summarized results for the domains considered using NumReach/SAT, NumReach/SMT and RANTANPLAN with the syntactic and the semantic notions of interference. For each domain we report the number of solved instances and their accumulated time steps of the commonly solved ones. We also show their averaged reductions in solving time and number of edges of the disabling graph.

\begin{tabular}{lc|ccc|ccc|cc}
\hline & & \multicolumn{3}{|c}{ Solved instances } & \multicolumn{3}{c}{ Accum. steps } & \multicolumn{3}{c}{ Averaged reductions } \\
& $\#$ & N/SAT & N/SMT & Sem. & N/SMT & Sem. & Syn. & Time & Edges \\
\hline Depots & 22 & 11 & 12 & 5 & 54 & 51 & 52 & $33.1 \%$ & $44.2 \%$ \\
Zenotravel & 20 & 13 & 14 & 13 & 97 & 68 & 68 & $22.0 \%$ & $70.8 \%$ \\
Petrobras & 15 & 6 & 8 & 8 & 69 & 65 & 65 & $30.2 \%$ & $59.1 \%$ \\
Planes & 12 & 4 & 8 & 9 & 170 & 116 & 154 & $84.3 \%$ & $86.8 \%$ \\
\hline
\end{tabular}

Moreover NumReach/SAT dominates NumReach/SMT in this domain. This happens because the numeric reasoning present in the domain is nearly null: the only functions present are for controlling load limits of trucks, and thus this domain is perfectly adequate for the approach used by NumReach/SAT. The use of a Linear Integer Arithmetic solver in the RANTANPLAN planner is overkill and a leaner and more efficient approach should be taken for problems of this kind. 


\section{Conclusions}

We have presented RANTANPlan, a new system for the setting of exact numeric planning. The planner is based on translation into SMT using a planning as satisfiability approach. It takes advantage of background theories in SMT to easily and transparently handle numeric fluents. Moreover it uses an SMT solver at compile time to detect in advance incompatibility between actions. This incompatibility results from lifting the interference notion of (Rintanen et al., 2006) to the setting of planning with resources. We have argued why the presented approach to interference between actions with numeric fluents is better than purely syntactically based ones, and provided empirical evidence of its usefulness. We have also shown that our system is competitive with the state of the art exact numeric planner NumReach.

\section{Acknowledgments}

All authors supported by the Spanish Ministry of Economy and Competitiveness through project HeLo (ref. TIN2012-33042) and project LoCoS (ref. TIN2015-66293-R).

\section{References}

Barrett, C., Sebastiani, R., Seshia, S., and Tinelli, C. (2009). Satisfiability Modulo Theories. In Handbook of Satisfiability, volume 185, chapter 26, pages 825-885. IOS Press.

Barták, R. and Toropila, D. (2010). Solving sequential planning problems via constraint satisfaction. Fundamenta Informaticae, 99(2):125-145.

Belouaer, L. and Maris, F. (2012). SMT Spatio-Temporal Planning. In ICAPS Workshop on Constraint Satisfaction Techniques for Planning and Scheduling Problems (COPLAS 2012), pages 6-15.

Bofill, M., Espasa, J., and Villaret, M. (2014). Efficient SMT Encodings for the Petrobras Domain. In Proceedings of the 13th International Workshop on Constraint Modelling and Reformulation (ModRef 2014), pages 68-84.

Bofill, M., Espasa, J., and Villaret, M. (2016). A Semantic Notion of Interference for Planning Modulo Theories. In Proceedings of the Twenty-Sixth International Conference on Automated Planning and Scheduling, ICAPS 2016, pages 56-64.

Dovier, A., Formisano, A., and Pontelli, E. (2010). Multivalued action languages with constraints in CLP (FD). Theory and Practice of Logic Programming, 10(02):167-235.

Dutertre, B. and De Moura, L. (2006). The Yices SMT Solver. Technical report, Computer Science Laboratory, SRI International. Available at http://yices.csl.sri.com.

Fox, M. and Long, D. (2003). PDDL2.1: An Extension to PDDL for Expressing Temporal Planning Domains. J. Artif. Intell. Res.(JAIR), 20:61-124.

Frisch, A. M. and Giannaros, P. A. (2010). SAT Encodings of the At-Most- $k$ Constraint. Some Old, Some New, Some Fast, Some Slow. In 10th International Workshop on Constraint Modelling and Reformulation (ModRef 2010).

Gerevini, A. E., Saetti, A., and Serina, I. (2008). An approach to efficient planning with numerical fluents and multi-criteria plan quality. Artificial Intelligence, 172(8):899-944.

Gregory, P., Long, D., Fox, M., and Beck, J. C. (2012). Planning Modulo Theories: Extending the Planning Paradigm. In Twenty-Second International Conference on Automated Planning and Scheduling (ICAPS 2012). AAAI.

Hoffmann, J. (2003). The Metric-FF Planning System: Translating "Ignoring Delete Lists" to Numeric State Variables. Journal of Artificial Intelligence Research, pages 291-341.

Hoffmann, J., Gomes, C. P., Selman, B., and Kautz, H. A. (2007). SAT Encodings of State-Space Reachability Problems in Numeric Domains. In 20th International Joint Conference on Artificial Intelligence (IJCAI 2007), pages 1918-1923.

Kautz, H. and Selman, B. (1992). Planning as Satisfiability. In 10th European Conference on Artificial Intelligence (ECAI 92), pages 359-363. John Wiley \& Sons, Inc.

Kautz, H. and Walser, J. P. (1999). State-space planning by integer optimization. In $A A A I / I A A I$, pages $526-533$.

Kautz, H. A., McAllester, D. A., and Selman, B. (1996). Encoding Plans in Propositional Logic. In Fifth International Conference on Principles of Knowledge Representation and Reasoning (KR 96), pages 374-384.

Rintanen, J. (2012). Planning as Satisfiability: Heuristics. Artificial Intelligence, 193:45-86.

Rintanen, J., Heljanko, K., and Niemelä, I. (2006). Planning as satisfiability: parallel plans and algorithms for plan search. Artificial Intelligence, 170(12-13):1031-1080.

Wolfman, S. A. and Weld, D. S. (1999). The LPSAT Engine \& Its Application to Resource Planning. In Sixteenth International Joint Conference on Artificial Intelligence (IJCAI 99), pages 310-317. 